\title{
Chemical Compatibility of Fused Filament Fabrication-based 3-D Printed Components with Solutions Commonly Used in Semiconductor Wet Processing
}

Ismo T. S. Heikkinen ${ }^{1}$, Christoffer Kauppinen ${ }^{1}$, Zhengjun Liu ${ }^{1}$, Sanja M. Asikainen ${ }^{2}$, Steven Spoljaric ${ }^{2}$ Jukka V. Seppälä ${ }^{2}$, Hele Savin ${ }^{1}$, Joshua M. Pearce ${ }^{1,3,4^{*}}$

1. Department of Electronics and Nanoengineering, School of Electrical Engineering, Aalto University, Espoo, Finland.

2. Department of Chemical and Metallurgical Engineering, School of Chemical Engineering, Aalto University, Espoo, Finland.

3. Department of Materials Science \& Engineering, Michigan Technological University, Houghton, MI USA.

4. Department of Electrical \& Computer Engineering, Michigan Technological University, Houghton, MI USA.

* Correspondence: pearce@mtu.edu ; Tel.: +906-487-1466

\begin{abstract}
3-D printing shows great potential in laboratories for making customized labware and reaction vessels. In addition, affordable fused filament fabrication (FFF)-based 3-D printing has successfully produced high-quality and affordable scientific equipment, focusing on tools without strict chemical compatibility limitations. As the additives and colorants used in 3-D printing filaments are proprietary, their compatibility with common chemicals is unknown, which has prevented their widespread use in laboratory chemical processing. In this study, the compatibility of ten widely available FFF plastics with solvents, acids, bases and solutions used in the wet processing of semiconductor materials is explored. The results provide data on materials unavailable in the literature and the chemical properties of 3-D printable plastics that were, are in line with literature. Overall, many 3-D printable plastics are compatible with concentrated solutions. Polypropylene emerged as a promising 3-D printable material for semiconductor processing due to its tolerance of strongly oxidizing acids, such as nitric and sulfuric acids. In addition, 3-D printed custom tools were demonstrated for a range of wet processing applications. The results show that 3-D printed plastics are potential materials for bespoke chemically resistant labware at less than $10 \%$ of the cost of such purchased tools. However, further studies are required to ascertain if such materials are fully compatible with clean room processing.
\end{abstract}

Keywords: 3-D printing; chemical compatibility; semiconductor wet processing; custom labware; clean rooms

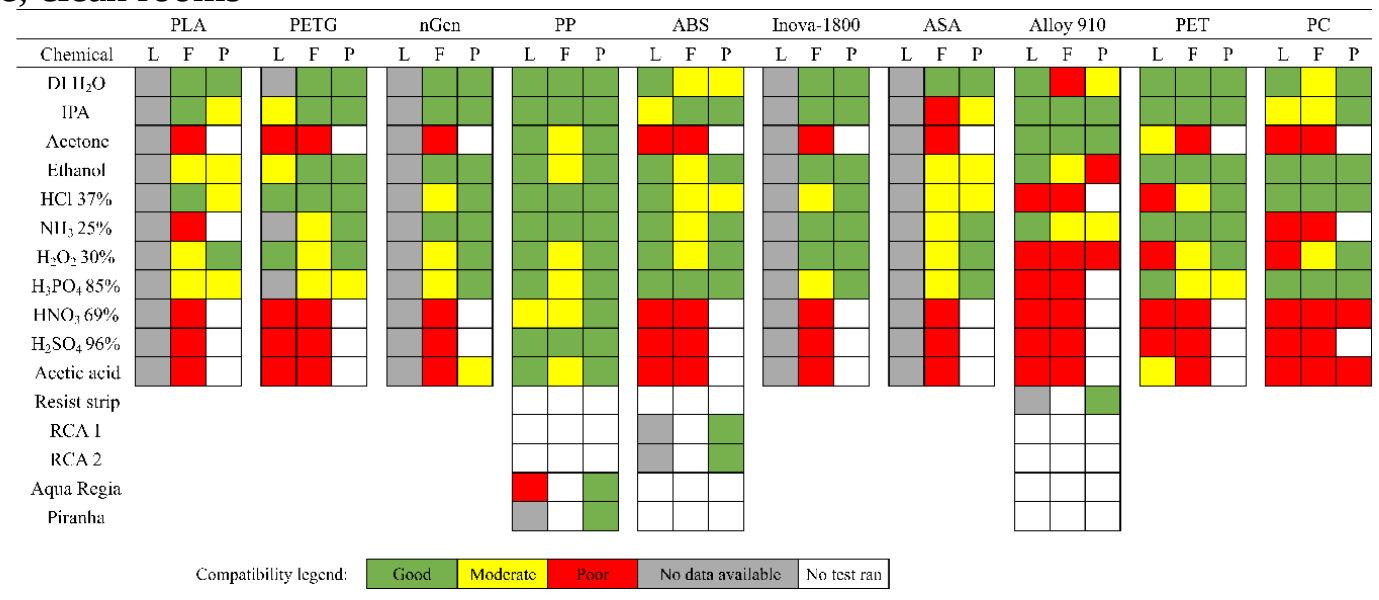




\section{Introduction}

3-D printing has shown considerable promise in chemical labs in the fabrication of chemical reactionware [1-4], millifluidics [5,6], microfluidics [7-10], and continuous flow chemistry [11]. In addition, accessible fused filament fabrication (FFF)-based 3-D printing [12-14] (also called fused deposition modeling (FDM) under trademark and material extrusion as stated in ISO/ASTM 52900:2017-02), has been shown to be effective at fabricating high-quality, bespoke, low-cost scientific equipment [15-17]. For example, digital re-creation of devices have been demonstrated for chemical mixing [18-20], biotechnological and chemical labware [20-24], colorimeters and turbidimeters [25-27], liquid autosamplers [28], and fluid handling [29-30], as well as mass spectroscopy equipment [31]. In general, this approach reduces capital cost of scientific equipment by 90-99\% compared to conventionally produced equipment [20,32], which has created substantial value for the scientific community [32]. This past work, however, has focused primarily on equipment without strict chemical compatibility standards or the use of known reagent-grade materials.

As the exact chemical formulation of low-cost commercial 3-D printing filaments (as well as additives such as plasticizers and colorants) is proprietary and thus chemical compatibility of printed parts is unknown, there has been no significant 3-D printing use in more challenging laboratory environments, such as those of clean rooms used for semiconductor processing. Due to the high cost of even basic equipment in clean rooms there is thus an opportunity to save funds while improving custom equipment with the use of 3-D printing. Furthermore, due to the high infrastructure and operation cost of the cleanroom, it is particularly important to improve the process throughput to fully utilize the facilities. In a multidiscipline researchorientated clean room and industrial R\&D facilities, significant time is spent on overcoming equipment limitations, because the equipment is installed to serve a wide range of research interests instead of the optimum for every process. Thus, 3-D printed accessories based on individual process requirements have the potential to improve cleanroom throughput as well as reducing processing costs by, for example, reducing the consumption of chemicals needed for processing. In order to exploit these opportunities, this study provides laboratory and cleanroom workers a materials toolkit for making their own reliable, customized, and chemically resistant labware from affordable materials using accessible FFF-based 3-D printers, as alternatives for commonly used polypropylene and fluoropolymer tools.

\section{Materials and Methods}

Specifically this study evaluates the chemical compatibility of 3-D printing polymers with wet chemical treatments commonly used in the clean room processing of semiconductor devices. We determine their chemical compatibility with common solvents and wet etchants using a down selection process. First, a range of commercial 3-D printing polymers is immersed in a range of common cleanroom chemicals for one week while monitoring mass and dimension changes after both surface and vacuum drying. These results are subsequently compared to chemical compatibility information available in the literature (chemical resistance charts from Curbell Plastics, Sirmax, Thermo Fisher Scientific and Plastics International) for the pure plastic that correlates to the main component of the filament. The 3-D printing materials considered are: polylactic acid (PLA), polyethylene terephthalate glycol (PETG), 2 different co-polyesters (Eastman Amphora AM3300-based nGen and Amphora 1800-based Inova1800), polypropylene (PP), acrylonitrile butadiene styrene (ABS), acrylonitrile styrene acrylate (ASA), polyamide copolymer-Nylon 6/69 (taulman3D Alloy 910), polyethylene 
terephthalate (PET, taulman3D t-glase), and polycarbonate (PC). The properties of the studied materials are summarized in Table 1.

Table 1. 3-D printing materials arranged by 3-D printing nozzle temperature.

\begin{tabular}{cccccc}
\hline Plastic & Product name & Supplier & Color & Cost $[\$ / k g]$ & Print $T\left[{ }^{\circ} \mathrm{C}\right]$ \\
\hline PLA & Polylite PLA & Polymaker & True Blue & 25 & 205 \\
PETG & PETG & Octofiber & Natural & 53 & 225 \\
$\begin{array}{c}\text { Eastman Amphora } \\
\text { 3300 }\end{array}$ & nGen & Colorfabb & Lulzbot green & 52 & 230 \\
$\begin{array}{c}\text { Eastman Amphora } \\
\text { 3300 }\end{array}$ & nGen & Colorfabb & Red & 52 & 230 \\
PP & PP & Ultimaker & Natural & 98 & 235 \\
ABS & ABS & IC3D & Green & 40 & 245 \\
Eastman Amphora & Inova-1800 & Chroma Strand & Blue & 80 & 245 \\
1800 & ASA Extrafill & Fillamentum & Traffic Black & 42 & 250 \\
ASA & Alloy 910 & taulman3D & Black & 79 & 255 \\
Polyamide copolymer- \\
Nylon 6/69
\end{tabular}

a) Price estimates are obtained from suppliers online stores specialized in 3-D printing.

The simple chemicals tested in the first phase are deionized water $\left(\mathrm{DI} \mathrm{H}_{2} \mathrm{O}\right)$, isopropyl alcohol (IPA), acetone, ethanol, hydrochloric acid ( $\mathrm{HCl}, 37 \%)$, ammonia $\left(\mathrm{NH}_{3}, 25 \%\right)$, hydrogen peroxide $\left(\mathrm{H}_{2} \mathrm{O}_{2}, 30 \%\right)$, phosphoric acid $\left(\mathrm{H}_{3} \mathrm{PO}_{4}, 85 \%\right)$, nitric acid $\left(\mathrm{HNO}_{3}, 69 \%\right)$, sulfuric acid $\left(\mathrm{H}_{2} \mathrm{SO}_{4}, 95-97 \%\right)$, and acetic acid (100\%). While many of the chemicals are generally used as dilute solutions, the 3-D printable plastics are immersed in concentrated solutions to study the upper limit of their chemical compatibility. If a plastic is compatible with a concentrated solution, it will most likely tolerate diluted solutions of the same chemicals for extended times. The studied chemicals are summarized in Table 2. Deionized water was obtained from the Micronova cleanroom water plant facilities (resistivity $>18 \mathrm{M} \Omega-\mathrm{cm}$ ). Other studied chemicals were obtained from commercial vendors (ethanol from Altia Plc / Technical Ethanol, Rajamäki, Finland, all others from Honeywell Specialty Chemicals Seelze GmbH, Seelze, Germany).

Table 2. Studied single chemicals, their formulae, concentrations and use in wet processing.

\begin{tabular}{cccc}
\hline Chemical & Formula & Concentration [\%] & Use \\
\hline Deionized water & $\mathrm{H}_{2} \mathrm{O}$ & - & Common solvent \\
2-propanol (isopropanol, IPA) & $\mathrm{CH}_{3} \mathrm{CHOHCH}_{3}$ & 100 & Organic solvent \\
Acetone & $\mathrm{CH}_{3} \mathrm{COCH}_{3}$ & 100 & Organic solvent \\
Ethanol & $\left(\mathrm{CH}_{3}\right) \mathrm{OCH}_{3}$ & 95 & Organic solvent \\
Hydrochloric acid & $\mathrm{HCl}$ & 37 & RCA 2, Aqua regia \\
Ammonia solution & $\mathrm{NH}_{3}$ & 25 & RCA 1 \\
Hydrogen peroxide & $\mathrm{H}_{2} \mathrm{O}_{2}$ & 30 & RCA 1, RCA 2 \\
Orthophosphoric acid & $\mathrm{H}_{3} \mathrm{PO}_{4}$ & 85 & Al etching
\end{tabular}




\begin{tabular}{cccc} 
Nitric acid & $\mathrm{HNO}_{3}$ & 69 & Si etch, Aqua regia \\
Sulfuric acid & $\mathrm{H}_{2} \mathrm{SO}_{4}$ & $95-97$ & Piranha \\
Acetic acid & $\mathrm{CH}_{3} \mathrm{COOH}$ & 100 & $\mathrm{Al}$ etching \\
Hydrofluoric acid & $\mathrm{HF}$ & 50 & $\mathrm{SiO}_{2}$ etch, cleaning \\
\hline
\end{tabular}

The experiments were divided into three phases: Phase 1 is the immersion of filaments in a single chemical, while in Phase 2 3-D printed rectangular pieces of the materials that showed acceptable compatibility were immersed in the same chemicals. Based on the results of Phases 1 and 2, suitable 3-D printable materials were selected for case studies based on their chemical tolerance and ease to print (e.g. no delamination and printable on a standard FFF type 3-D printer with no alterations). Printed samples that pass the tests are analyzed with differential scanning calorimetry (DSC) to evaluate changes in the crystallinity, glass transition and melting temperatures of the polymers, which are closely linked to, amongst other factors, polymer chain structure, repeating unit type and length, molecular weight, branching, additives, etc. [33,34]. The materials that withstand the simple chemical environments are then tested in more demanding chemical processes and solutions commonly used in semiconductor device fabrication: photoresist strip, RCA 1 and RCA 2 cleaning, hydrogen fluoride (HF) dip, Aqua regia immersion, and Piranha immersion [35]. In addition, in Phase 3 open source parametric 3-D printable laboratory tools were immersed in the solutions. In the down selection process, a $\pm 5 \%$ change in mass or dimensions of the sample was used as the acceptance criterion, but in determining the practical compatibility of the polymers with specific solutions a stricter $\pm 1 \%$ criterion was considered.

Samples for Phase 1 were prepared from FFF materials as received from the manufacturer. All samples were handled with nitrile gloves to protect the filaments from contaminants resulting from skin contact. 20-25 mm long strands of filament were cut from the spools using conventional scissors cleaned with IPA. All individual pieces of filament were weighed with a VWR (Radnor, PA, USA) precision scale and their diameters were measured using a digital caliper with an accuracy of $\pm 0.01 \mathrm{~mm}$. After preparation, the samples were placed into borosilicate glass vials (clear Wheaton $4 \mathrm{ml}$ vials with styrene-butadiene caps) using IPAcleaned plastic tweezers (NKR RT, Outils Rubis SA, Stabio, Switzerland). Approximately 50 $\mathrm{ml}$ of a chemical was poured into a beaker (VWR, PP, $100 \mathrm{ml}$ ), which had been first rinsed with water and the chemical in use, and a sufficient amount of the chemical was dispensed to the vials using plastic pipettes (VWR, LD-PE) to fully immerse the samples. The caps of the vials were tightly closed and the vials were preserved in a closed opaque polypropylene container inside a fume hood for one week. The caps of the nitric and sulfuric acid vials were not closed as the strong acids could have reacted with the plastics and caused pressure to build up inside the glass vial. Common immersion times in these solutions are normally short in semiconductor processing ranging from minutes to tens of minutes. An immersion time of one week was used in Phases 1 and 2 to ensure the 3-D printed objects would be adequately chemically resistant for all normal use scenarios. The temperature of the solutions was not controlled in Phases 1 and 2, the samples were kept inside a fume hood in room temperature. After the immersion, the caps of the vials were opened, and the samples were picked up from the solutions using similar water-rinsed plastic tweezers and rinsed twice in deionized water for 20s. The pieces of filament were surface dried with a $\mathrm{N}_{2}$ gun before weighing them with the same scales as before immersion. After weighing, the diameter of the surface dried samples was measured again using the same digital caliper. The "surface dried" condition was used as the eliminator: if the filament had swollen in the immersion, it was not compatible with the chemical. $\pm 5 \%$ of change in mass or diameter of the filament was used as the acceptance criterion in the down selection process, and compatible materials were selected for Phase 2. 
To make sure the thermoplastic extrusion printing does not impair the chemical durability of the plastics, also 3-D printed samples of the same materials were subjected to similar chemical immersion tests. The 3-D printed samples were only subjected to the chemicals that did not damage the filaments in Phase 1. Rectangular objects with 100 percent infill were printed with the same spools of material using a Lulzbot Taz 6 printer (Aleph Objects, Loveland CO). After printing, all samples were handled with gloves. The sample dimensions were $23 \mathrm{~mm}$ x $6 \mathrm{~mm}$ x 2mm [36], and all printing parameters are described in detail in Table 3 . The printer and slicing settings were defined using Cura 21.08 Lulzbot edition slicing software. For certain samples (nGen, Alloy 910, Inova-1800, PET, and PC) a PVA-based glue was applied to the printing bed in the fabrication to decrease the samples' adhesion to the printing bed. For PP, clear PP-based packing tape was used to promote the bed adhesion. The residual glue and packing tape were removed from all the samples by scraping them with a knife and wiping them repeatedly with water- and IPA-immersed cleanroom wipes. Right before immersion all 3-D printed samples were wiped clean with a cleanroom cloth immersed in IPA.

Table 3. Essential Cura settings for printing the test samples used in this study.

\begin{tabular}{cccccccccccc}
\hline Setting & Units & PLA & PETG & nGen & Alloy 910 & PP & PET & ABS & Inova & ASA & PC \\
\hline Layer Height & $\mathrm{mm}$ & 0.18 & 0.18 & 0.18 & 0.25 & 0.18 & 0.45 & 0.18 & 0.15 & 0.1 & 0.15 \\
Shell Thickness & $\mathrm{mm}$ & 1.0 & 1.0 & 1.0 & 3.0 & 1.0 & 1.8 & 1.0 & 1.0 & 1.0 & 1.0 \\
Bottom/Top & $\mathrm{mm}$ & 1.08 & 1.08 & 1.08 & 2.0 & 1.08 & 1.35 & 1.08 & 1.05 & 1.0 & 0.9 \\
Thickness & & & & & & & & & & & \\
Print Speed & $\mathrm{mm} / \mathrm{s}$ & 50 & 30 & 50 & 30 & 50 & 20 & 50 & 50 & 37 & 50 \\
Top/Bottom & $\mathrm{mm} / \mathrm{s}$ & 45 & 15 & 10 & 30 & 10 & 10 & 30 & 20 & 45 & 30 \\
$\quad$ Speed & & & & & & & & & & & \\
Print $T$ & ${ }^{\circ} \mathrm{C}$ & 205 & 225 & 230 & 255 & 235 & 255 & 245 & 245 & 250 & 255 \\
$\quad$ Bed $T$ & ${ }^{\circ} \mathrm{C}$ & 60 & 55 & 85 & 100 & 60 & 60 & 95 & 80 & 100 & 100 \\
Minimal Layer & $\mathrm{sec}$ & 30 & 14 & 15 & 15 & 15 & 20 & 15 & 5 & 15 & 20 \\
Time & & & & & & & & & & & \\
\hline
\end{tabular}

The 3-D printed samples were immersed similarly as the virgin filaments in Phase 1, but after the immersion, surface drying, and weighing and measuring their dimensions the samples were left to dry inside a fume hood in air overnight. After drying in air, the printed samples were vacuum dried in a SalvisLab VC20 vacuum oven for at least 4 days to make sure that all the absorbed solution would have evaporated from the filaments. Promising 3-D printed samples were further characterized with DSC using a TA Instruments Q2000 DSC tool to observe possible changes in the crystallinity, glass transition and melting temperatures of the samples. The vacuum drying was used to evacuate all absorbed solvent from the samples before the DSC measurement: if there were solvent absorbed into the polymer, it might have boiled and broken the sample container during the heating cycles. Specimens (5-11mg) were heated at $20^{\circ} \mathrm{C} / \mathrm{min}$, in order to remove their thermal history. This was followed by a cooling cycle to at $20^{\circ} \mathrm{C} / \mathrm{min}$ and a second heating cycle at $20^{\circ} \mathrm{C} / \mathrm{min}$. Transition temperatures and enthalpy of melting were recorded from the results of the second heating cycle. The upper and lower limits of the DSC runs are presented in Table 4. 
Table 4. Temperature range of DSC runs.

\begin{tabular}{ccc}
\hline Material & Lower limit $\left[{ }^{\circ} \mathrm{C}\right]$ & Upper limit $\left[{ }^{\circ} \mathrm{C}\right]$ \\
\hline PP & -70 & 180 \\
ABS & -90 & 270 \\
Alloy 910 & 0 & 290 \\
PC & 0 & 300 \\
\hline
\end{tabular}

Suitable materials for Phase 3 were selected based on the results from Phase 2. Figure 1 presents the protocols of the wet processing case studies. In these experiments HF dipping and Piranha immersion differed slightly from the industry standards described above.

Photoresist stripping refers to removal of photoresist from wafers after the resist has been used in patterning the substrate. A common way to achieve this is to immerse the wafer in acetone and place them into an ultrasonic bath, which effectively dissolves the resist providing that the resist has not been damaged by plasma or ion bombardment during the patterning. After sonication the wafer can be further immersed in acetone and isopropanol and rinsed with deionized water to make sure all resist has been removed [35].

In semiconductor processing wafers are often cleaned prior to process steps by RCA 1 and RCA 2 cleaning methods. RCA 1 solution consists of ammonia and hydrogen peroxide, and it removes organic contaminants from the wafers, while RCA 2 solution, consisting of hydrochloric acid and hydrogen peroxide, removes metal contaminants. Both cleaning methods are performed at elevated temperature, up to $80^{\circ} \mathrm{C}$, and typical cleaning duration ranges from 10 to $20 \mathrm{~min}$. In industry dilute solutions are often used [35].

Hydrofluoric acid is commonly used in semiconductor processing to remove silicon oxide from the surface of silicon wafers, which promotes the removal of contaminants from the substrate after RCA cleans. Generally very dilute HF solutions are used, ranging from 1:20 to 1:500 $\mathrm{HF}: \mathrm{H}_{2} \mathrm{O}$ ratios, and usually 1 min HF immersion is enough to remove thin oxides from Si surface. Exposure to HF can be fatal and a separate system is needed for its disposal, so the chemical needs to be handled with particular care [35].

Aqua regia is commonly used in the wet etching of metals [37]. Aqua regia is a mix of $\mathrm{HCl}$ and $\mathrm{HNO}$, often used in a 4:1 $\mathrm{HCl} 37 \%$ : HNO3 69\% volumetric ratio. Specifically, Aqua regia is used to remove even noble metals like $\mathrm{Au}$ and $\mathrm{Pt}[38,39]$. Aqua regia is also used as a semiconductor surface treatment for example in obtaining low contact resistance p-GaN [40,41], which is important for high efficiency white LEDs and blue lasers.

Piranha solution is widely used as a facile method to remove organic contamination [42] and to remove photoresist residues from silicon wafers [43]. Piranha is a mixture of $\mathrm{H}_{2} \mathrm{SO}_{4} 96 \%$ and $\mathrm{H}_{2} \mathrm{O}_{2} 30 \%$ in a $4: 1$ volumetric ratio. It is often used at $120^{\circ} \mathrm{C}$ with typical immersion time ranging from 10 to $20 \mathrm{~min}$ [35]. The mixing of the Piranha solution is very exothermic and should be done with all the necessary chemical safety procedures and care, as mixing the solution alone heats the liquid up to $100^{\circ} \mathrm{C}$. 


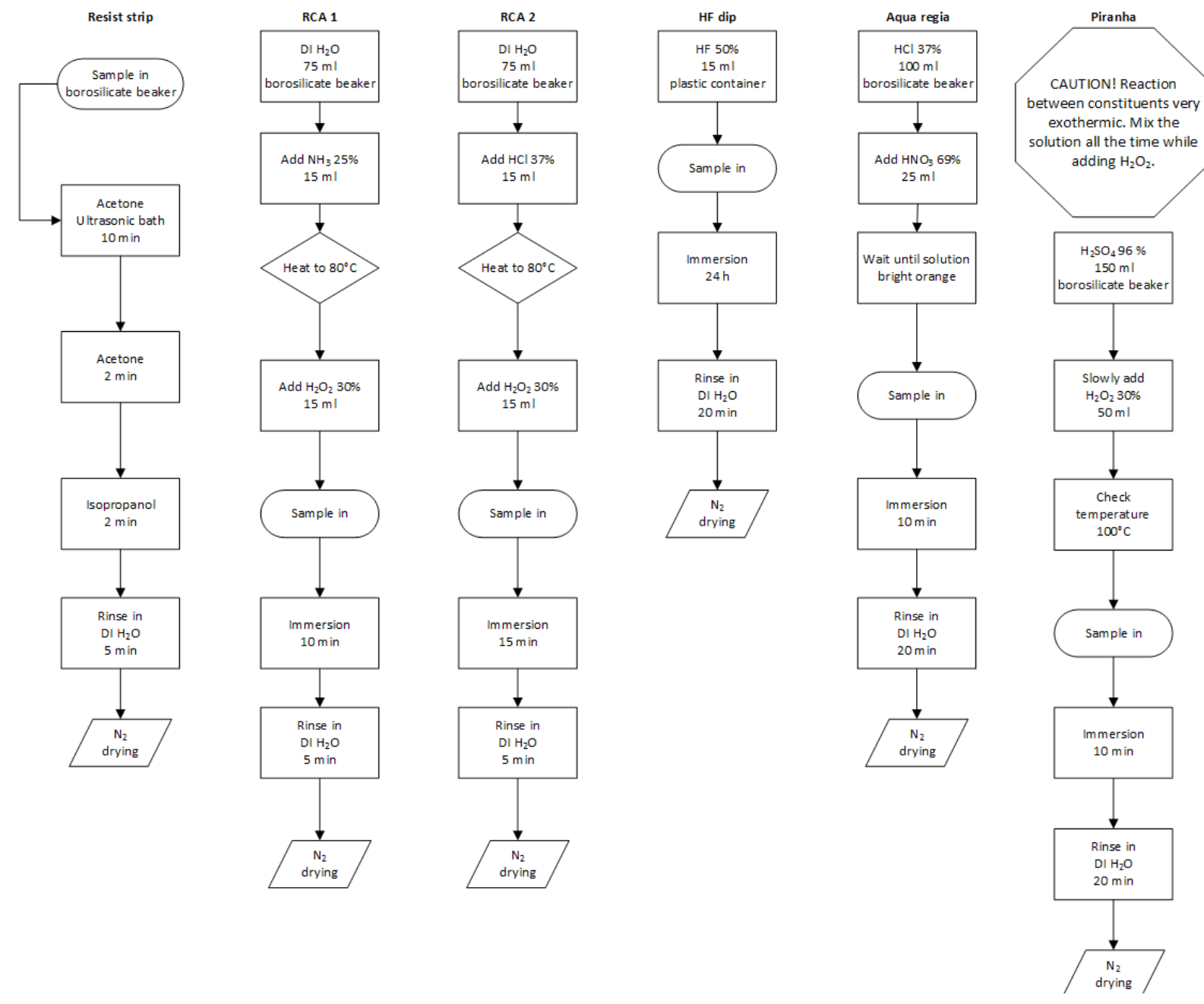

Figure 1. Studied chemical processes and immersions with temperatures and associated conditions: photoresist stripping, RCA 1 and 2, Aqua regia, HF and Piranha immersions. 


\section{Results and Discussion}

Mass and diameter change results measured from pieces of filament in Phase 1 and rectangular 3-D printed objects in Phases 2 and 3 after surface drying are presented in Table 5 and DSC results from PP, ABS and Alloy 910 samples from Phases 2 and 3 are shown in Table 6. Virgin filaments were generally less resistant to the solutions than the 3-D printed samples. All materials tolerated the one-week immersion in deionized water and isopropanol, but only PP and nylon-based Alloy 910 showed resistance to acetone. However, as water can hydrolyze PLA and plasticize nylon, their long-term stability in aqueous solutions is questionable. ASA first appeared to tolerate ethanol well, but the sample was flexible immediately after immersion, regaining its rigidity after vacuum drying. Many materials had good resistance to $\mathrm{HCl}$ and $\mathrm{H}_{3} \mathrm{PO}_{4}$, but nitric, sulfuric, and acetic acids destroyed most plastics. Polypropylene survived them all. $\mathrm{HNO}_{3}$ and $\mathrm{H}_{2} \mathrm{SO}_{4}$ slightly colored the surface of PP: In nitric acid, PP samples turned slightly yellow and in sulfuric acid small brown areas appeared on the sample. This discoloration suggests that oxidation may have occurred and must be considered if extended immersion times within the aforementioned acids is desired. However, no changes in the mass, dimensions, or thermal properties in those samples were observed.

Based on the initial experiments, three materials were chosen for tests in more realistic chemical case studies used in semiconductor processing. The printed materials were chosen for each immersion based on their chemical resistance, availability, and ease to print. Alloy 910, which tolerated water, IPA and acetone quite well, was used for resist stripping. ABS was selected for RCA cleaning due to its good thermal properties and resistance to hydrochloric acid, ammonia, and hydrogen peroxide, but also because it is one of the most common 3-D printable plastics. PP was the material of choice for Aqua regia, HF and Piranha immersions. Customized labware [36] was designed, printed, and immersed into the solutions with rectangular monitor samples.

For the photoresist stripping tweezers [44] were printed out of Alloy 910 and immersed in the solvents. For RCA cleanings as well as Aqua Regia and Piranha immersions a parametric 2 inch wafer dipper was designed and printed out of ABS for RCA cleaning (with a modified handle) and of PP for the other immersions. In all immersions, a similar rectangular sample as used in the previous studies was used to monitor if the solutions affected the employed polymer in any way. All printed objects tolerated the immersions well: there was no significant change in mass or dimensions for any small sample, and the tools were intact and functional after immersion. The DSC results did not show any differences compared to samples immersed in the simple chemicals of each study. Not even the Piranha solution significantly affected printed polypropylene. Examples of the case studies are shown in Figure 2. In addition to the dipper, a replacement handle for a standard 3 inch wafer jig was designed and printed out of PP, shown in Figure 2f, to demonstrate that 3-D printing is viable option for fabricating spare parts for existing laboratory equipment.

Selected samples studied in Phases 2 and 3 were further characterized with DSC to confirm that the solutions do not damage the polymers in the 3-D printable materials. Glass transition temperature $\left({ }^{\circ} \mathrm{C}\right)$ and crystallinity values of PP, ABS, and Alloy 910. ABS displayed subtle variations in $\mathrm{T}_{\mathrm{g}}$ for all test specimens, highlighting its stability and minimal influence of solvents on material structure. PP displayed small fluctuations in $\mathrm{T}_{\mathrm{g}}$ following treatments, with slight reductions in crystallinity values compared with the pure specimen. Exposure to $\mathrm{H}_{2} \mathrm{O}_{2}$, HF and Piranha solution lead to the greatest reduction in crystallinity of PP, in the range of $\sim 3-4 \%$. Alloy 910 experienced the most drastic change in glass transition temperatures, 
dropping from 75 (pure specimen) to $54^{\circ} \mathrm{C}$ (acetone immersion). Similarly, all other Alloy 910 specimens immersed in solvent displayed a drop in $\mathrm{T}_{\mathrm{g}}$. Additionally, the enthalpy of melting dropped for all immersed samples, suggesting a reduction in crystallinity. This is indicative of the ability of nylon's amide linkages to readily absorb a range of solvents, especially water, alcohols and acetone. Bound solvent molecules can cause swelling within the network, increasing free volume which results in a plasticizing effect (lower $T_{g}$ values). This also significantly increases chain mobility which may result in reduced crystallinity values. With the exception of Alloy 910, both ABS and PP displayed clear solvent stability, experiencing negligible changes in glass transition and melting temperatures and crystallinity.

With the exception of Alloy 910, negligible changes in crystallinity and glass transition were observed for the remaining polymer specimens, while melting temperature remained quite constant for all specimens. Generally, it seems that the additives do not affect the chemical properties of the commercial 3-D printable materials compared to base plastics, and that the thermoplastic extrusion does not make the plastic chemically less-resistant.

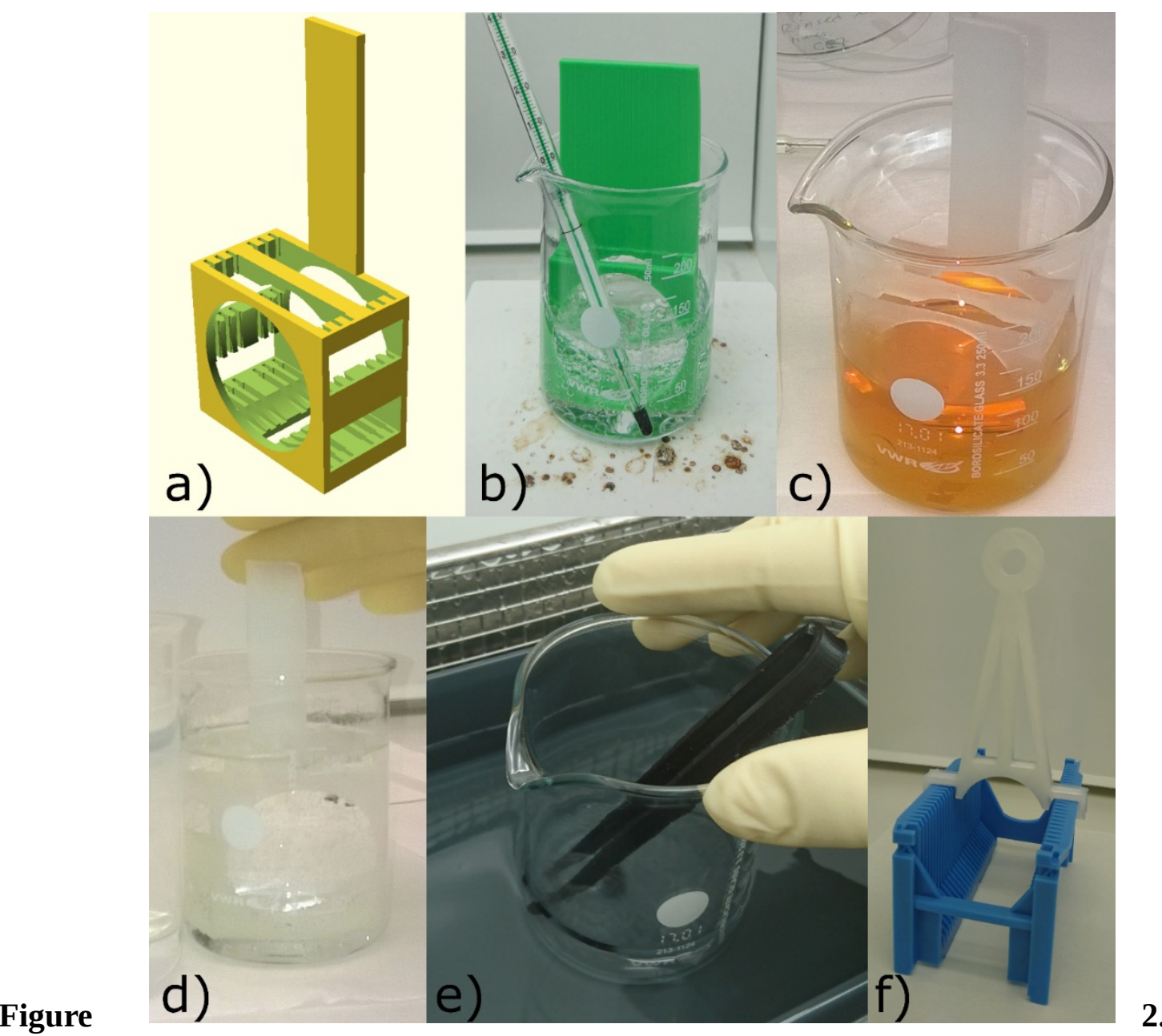

Examples of 3-D printed labware: a) 3-D model of the 2 inch dipper, b) modified dipper made of green ABS in RCA 1 solution in a borosilicate beaker, c) clear PP dipper in Aqua regia solution, d) PP dipper immersed into Piranha, e) Black Alloy 910 tweezers in sonicated acetone, and f) a large clear PP handle for a 3 inch wafer jig. 
Table 5. Mass and diameter changes for all materials tested in Phases 1, 2 and 3.

\begin{tabular}{|c|c|c|c|c|c|c|c|c|c|c|c|c|}
\hline Chemical & & $\Delta[\%]$ & PLA & PETG & nGen & PP & ABS & Inova & ASA & Alloy 910 & PET & PC \\
\hline \multirow{4}{*}{ DI $\mathrm{H}_{2} \mathrm{O}$} & \multirow{2}{*}{$\mathrm{F}$} & $\Delta m$ & 0.0 & 0.0 & 0.0 & 0.0 & 0.0 & 0.4 & 0.5 & 5.1 & 0.5 & 0.9 \\
\hline & & $\Delta d$ & 0.7 & 0.0 & 0.0 & 0.0 & 1.4 & -0.7 & 0.0 & 1.7 & 0.4 & 1.1 \\
\hline & \multirow{2}{*}{$P$} & $\Delta m$ & 0.3 & 0.3 & -0.3 & 0.0 & 0.0 & -0.3 & -0.3 & 4.3 & 0.3 & -0.3 \\
\hline & & $\Delta d$ & 0.0 & 0.0 & -0.1 & 0.1 & -1.9 & 0.3 & 0.3 & 0.4 & 0.7 & -0.1 \\
\hline \multirow{4}{*}{ IPA } & \multirow{2}{*}{$\mathrm{F}$} & $\Delta m$ & 0.0 & 0.0 & 0.0 & 0.0 & 0.5 & 0.0 & 5.3 & 0.0 & 0.0 & 0.5 \\
\hline & & $\Delta d$ & 0.7 & 0.4 & 0.4 & 0.4 & 0.0 & -0.4 & 2.5 & 0.3 & 0.0 & 1.1 \\
\hline & \multirow{2}{*}{$P$} & $\Delta m$ & 0.3 & -0.3 & -0.3 & -0.4 & 0.0 & -0.3 & 2.0 & 0.4 & 0.0 & -0.3 \\
\hline & & $\Delta d$ & 1.7 & -0.4 & 0.0 & -0.5 & -0.1 & -0.2 & 0.1 & 0.2 & -0.5 & 0.0 \\
\hline \multirow{4}{*}{ Acetone } & \multirow{2}{*}{$\mathrm{F}$} & $\Delta m$ & -100 & 17.6 & 11.5 & 2.4 & -100 & 17.3 & -100 & -0.5 & 17.2 & 16.7 \\
\hline & & $\Delta d$ & -100 & 6.9 & 4.6 & 1.1 & -100 & 5.3 & -100 & 0.7 & 2.9 & 20.4 \\
\hline & \multirow{2}{*}{$P$} & $\Delta m$ & & & 8.2 & 0.9 & & & & 0.0 & & \\
\hline & & $\Delta d$ & & & 3.9 & 0.3 & & & & -0.2 & & \\
\hline \multirow{4}{*}{ Ethanol } & \multirow{2}{*}{$\mathrm{F}$} & $\Delta m$ & 1.3 & -0.4 & 0.0 & -1.3 & 1.6 & -0.4 & 3.0 & 2.7 & 0.0 & 0.0 \\
\hline & & $\Delta d$ & 0.7 & 0.0 & 0.7 & 0.0 & 1.1 & 0.0 & 3.6 & 1.4 & 0.4 & 0.4 \\
\hline & \multirow{2}{*}{$P$} & $\Delta m$ & 1.9 & 0.5 & 0.7 & 0.4 & 0.7 & 0.6 & 3.0 & 8.7 & 0.0 & 0.3 \\
\hline & & $\Delta d$ & -0.5 & -0.2 & 0.4 & 0.1 & -0.1 & -0.2 & 0.8 & 3.0 & -0.4 & -0.3 \\
\hline \multirow{4}{*}{$\mathrm{HCl}$} & \multirow{2}{*}{$F$} & $\Delta m$ & 0.0 & -0.5 & -0.4 & 0.0 & 1.6 & -0.4 & 2.0 & -100 & 0.0 & 0.0 \\
\hline & & $\Delta d$ & 0.4 & 0.7 & 1.8 & 0.4 & 2.5 & 1.1 & 0.0 & -100 & -1.4 & 0.7 \\
\hline & \multirow{2}{*}{$P$} & $\Delta m$ & 0.8 & 0.5 & 0.0 & -0.4 & 1.7 & 0.3 & 2.3 & & 0.6 & 0.3 \\
\hline & & $\Delta d$ & 1.9 & 0.0 & -0.1 & 0.1 & 0.3 & -0.3 & 0.6 & & 0.0 & -0.3 \\
\hline & & $\Delta m$ & -84 & -0.4 & 0.9 & 0.0 & 1.1 & 0.0 & 1.0 & 1.5 & 0.0 & -15.9 \\
\hline & $F$ & $\Delta d$ & -100 & 1.4 & 0.4 & 0.7 & 1.0 & 0.4 & 0.7 & 1.0 & 0.0 & -100 \\
\hline $\mathrm{NH}_{3}$ & P & $\Delta m$ & & 0.0 & 0.3 & 0.0 & 0.3 & 0.3 & 0.0 & 1.4 & 0.0 & \\
\hline & $P$ & $\Delta d$ & & 0.4 & -0.5 & 0.3 & 0.1 & 0.3 & -0.1 & 0.7 & 0.5 & \\
\hline & & $\Delta m$ & 1.0 & 1.2 & 0.0 & 1.8 & 1.1 & 0.5 & 1.0 & 19.9 & 1.5 & 0.0 \\
\hline & F & $\Delta d$ & 1.1 & 1.8 & 1.4 & 1.4 & 2.0 & 0.0 & 1.4 & 4.2 & 1.5 & 1.4 \\
\hline${ }_{2} \mathrm{O}_{2}$ & D & $\Delta m$ & 0.8 & 0.5 & 0.3 & 0.0 & 0.7 & 0.3 & 0.0 & 12.6 & 0.3 & 0.0 \\
\hline & $P$ & $\Delta d$ & 0.2 & -0.1 & -0.5 & -0.5 & 0.5 & -0.1 & 0.0 & 3.0 & -0.1 & 0.2 \\
\hline & 5 & $\Delta m$ & 1.0 & 1.7 & 0.5 & 1.3 & 0.0 & 0.4 & 2.7 & -100 & 0.4 & 0.5 \\
\hline & F & $\Delta d$ & 1.8 & 1.4 & 1.1 & 0.4 & 0.7 & 1.4 & 1.1 & -100 & 1.1 & -0.4 \\
\hline $\mathrm{H}_{3} \mathrm{PO}_{4}$ & D & $\Delta m$ & 2.2 & 3.0 & 0.3 & 0.0 & -0.3 & 0.0 & 0.3 & & 1.1 & 0.0 \\
\hline & $P$ & $\Delta d$ & -0.4 & -0.4 & -0.3 & 0.3 & 0.3 & 0.1 & 0.6 & & 0.8 & -0.3 \\
\hline & $\mathrm{F}$ & $\Delta m$ & -100 & -100 & 39.3 & 1.2 & -100 & -100 & 127 & -100 & -100 & 10.1 \\
\hline & F & $\Delta d$ & -100 & -100 & 15.7 & 1.1 & -100 & -100 & -100 & -100 & -100 & 6.1 \\
\hline $\mathrm{HNO}_{3}$ & P & $\Delta m$ & & & & 0.0 & & & & & & 8.5 \\
\hline & $P$ & $\Delta d$ & & & & 0.0 & & & & & & 0.9 \\
\hline & 5 & $\Delta m$ & -100 & -100 & -100 & -0.6 & -100 & -100 & -100 & -100 & -100 & -100 \\
\hline $\mathrm{HSO}$ & $r$ & $\Delta d$ & -100 & -100 & -100 & 0.0 & -100 & -100 & -100 & -100 & -100 & -100 \\
\hline $\mathrm{H}_{2} \mathrm{SO}_{4}$ & D & $\Delta m$ & & & & 0.0 & & & & & & \\
\hline & $P$ & $\Delta d$ & & & & 0.7 & & & & & & \\
\hline & $\mathrm{F}$ & $\Delta m$ & 61.0 & 19.8 & 6.5 & 4.3 & 75.0 & 17.4 & -100 & 67.9 & 17.5 & 26.4 \\
\hline Aretic acid & $r$ & $\Delta d$ & 18.5 & 6.1 & 2.1 & 1.1 & -100 & 4.2 & -100 & 18.5 & 2.2 & 7.5 \\
\hline Aсецс асіа & $P$ & $\Delta m$ & & & 4.3 & 0.9 & & & & & & 14.1 \\
\hline & $P$ & $\Delta d$ & & & 0.7 & 0.1 & & & & & & 6.9 \\
\hline Rocict ctrin & $\mathrm{P}$ & $\Delta m$ & & & & & & & & -0.4 & & \\
\hline Resist strıp & $\mathrm{P}$ & $\Delta d$ & & & & & & & & 0.4 & & \\
\hline $\mathrm{PCA} 1$ & $P$ & $\Delta m$ & & & & & 0.7 & & & & & \\
\hline RCA 1 & $\mathrm{P}$ & $\Delta d$ & & & & & 0.4 & & & & & \\
\hline DCA & D & $\Delta m$ & & & & & 0.7 & & & & & \\
\hline RCA 2 & $P$ & $\Delta d$ & & & & & -0.1 & & & & & \\
\hline HE din & P & $\Delta m$ & & & & 0.9 & & & & & & \\
\hline HF dıp & $\mathrm{P}$ & $\Delta d$ & & & & 0.2 & & & & & & \\
\hline regia & D & $\Delta m$ & & & & 0.9 & & & & & & \\
\hline & $P$ & $\Delta d$ & & & & -0.2 & & & & & & \\
\hline Piranha & $P$ & $\begin{array}{l}\Delta m \\
\Delta d\end{array}$ & & & & $\begin{array}{l}0.0 \\
-0.2\end{array}$ & & & & & & \\
\hline
\end{tabular}


Table 6: Glass transition temperature $T_{g}\left[{ }^{\circ} \mathrm{C}\right]$, melting temperature $T_{m}\left[{ }^{\circ} \mathrm{C}\right]$ and crystallinity values of printed samples as-printed and after chemical immersions.

\begin{tabular}{|c|c|c|c|c|}
\hline Plastic & Immersion & $T_{g}\left[{ }^{\circ} \mathrm{C}\right]$ & $\begin{array}{c}\text { Crystallinity [\%] }]^{\mathrm{a})} / \\
\text { Enthalpy of melting [J/g] }\end{array}$ & $T_{m}\left[{ }^{\circ} \mathrm{C}\right]$ \\
\hline \multirow{15}{*}{ PP } & As-printed & -22 & 15 & 134 \\
\hline & DI $\mathrm{H}_{2} \mathrm{O}$ & -23 & 15 & 131 \\
\hline & Isopropanol & -23 & 15 & 134 \\
\hline & Acetone & -24 & 13 & 133 \\
\hline & Ethanol & -23 & 15 & 133 \\
\hline & $\mathrm{HCl} 37 \%$ & -23 & 14 & 134 \\
\hline & $\mathrm{NH}_{3} 25 \%$ & -21 & 15 & 134 \\
\hline & $\mathrm{H}_{2} \mathrm{O}_{2} 30 \%$ & -21 & 15 & 134 \\
\hline & $\mathrm{H}_{3} \mathrm{PO}_{4} 85 \%$ & -23 & 15 & 131 \\
\hline & $\mathrm{HNO}_{3} 69 \%$ & -22 & 14 & 134 \\
\hline & $\mathrm{H}_{2} \mathrm{SO}_{4} 95-97 \%$ & -23 & 15 & 132 \\
\hline & Acetic acid 100\% & -21 & 15 & 134 \\
\hline & HF dip & -20 & 14 & 135 \\
\hline & Aqua regia & -23 & 14 & 134 \\
\hline & Piranha & -22 & 14 & 134 \\
\hline \multirow{9}{*}{ ABS } & As-printed & 104 & - & - \\
\hline & $\mathrm{DI} \mathrm{H}_{2} \mathrm{O}$ & 102 & - & - \\
\hline & Isopropanol & 103 & - & - \\
\hline & $\mathrm{HCl} 37 \%$ & 106 & - & - \\
\hline & $\mathrm{NH}_{3} 25 \%$ & 104 & - & - \\
\hline & $\mathrm{H}_{2} \mathrm{O}_{2} 30 \%$ & 104 & - & - \\
\hline & $\mathrm{H}_{3} \mathrm{PO}_{4} 85 \%$ & 104 & - & - \\
\hline & RCA 1 & 105 & - & - \\
\hline & RCA 2 & 105 & - & - \\
\hline \multirow{5}{*}{ Alloy 910} & As-printed & 75 & 43.2 & 196 \\
\hline & DI $\mathrm{H}_{2} \mathrm{O}$ & 68 & 36.2 & 195 \\
\hline & Isopropanol & 56 & 36.16 & 196 \\
\hline & Acetone & 54 & 41.35 & 195 \\
\hline & Resist strip & 55 & 37.38 & 195 \\
\hline
\end{tabular}

a) Only PP and Alloy 910 are semi-crystalline, ABS is amorphous. ${ }^{\text {b) }}$ Due to the exotic nature of Alloy 910, an enthalpy of melting for a $100 \%$ crystalline specimen is not available and thus crystallinity cannot be determined using DSC. Therefore, the enthalpy of melting values are listed. 
Figure 3 summarizes the results of the chemical immersion experiments and compares them to the literature. The leftmost column represents reported compatibility of the polymer with the chemical in the literature $(\mathrm{L})$, while the center and the rightmost column present the results from chemical immersion tests for pure filaments $(\mathrm{F})$ and 3-D printed $(\mathrm{P})$ samples in this study, respectively. Literature compatibility has been assessed by comparing different chemical compatibility charts published by polymer and labware manufacturers [45-49]. While the compatibilities reported in the literature likely apply for materials that structurally differ from the 3-D printable materials, they are used as general guidelines to assess the chemical resistance of the main polymers of the filaments. In determining the practical compatibility of the polymers with specific solutions, $\pm 1 \%$ change in mass or dimensions was considered as the acceptance criterion. Green color signifies that the polymer does not dissolve, absorb solvent, or swell during the one-week immersion (change in mass and dimensions below 1\%), while yellow indicates that some swelling or mass change occurred (change in mass or dimensions 2-5\%). Red color signifies significant mass change, heavy swelling (change in mass or dimensions above $5 \%$ ) or complete destruction of the filament.

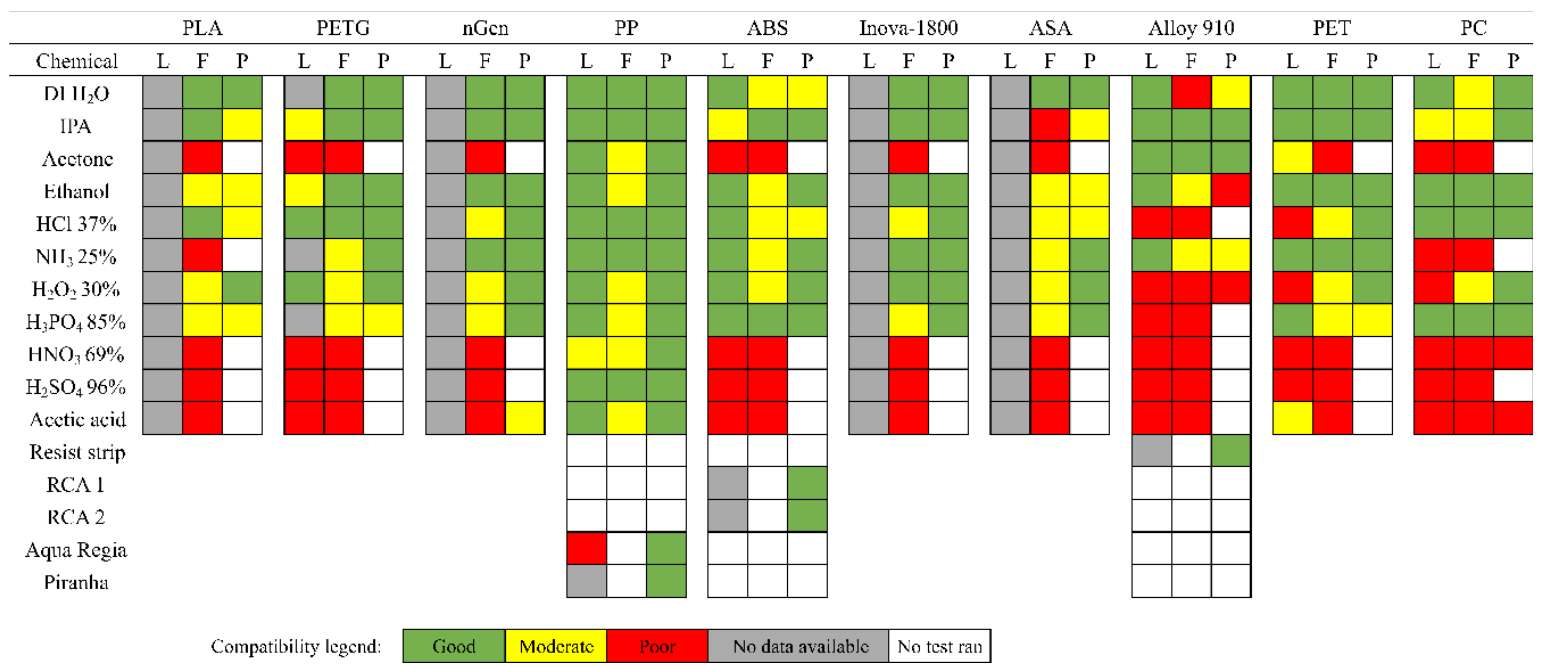

Figure 3. Map of chemical compatibility of plastics and 3-D printing materials from the literature (L) [45-49], as virgin filament (F) and 3-D printed (P). 


\section{Discussion}

The results for the materials for which compatibilities have been reported in the literature were mostly in line with the reported compatibility. Therefore, the additives and antioxidants used in 3-D printing filaments do not significantly impair their chemical properties compared to virgin polymers. All case studies were successful showing no loss of dimensional stability from the relatively extreme chemical environments. 3-D printed PP emerged as the most promising material for various chemical environments, but also cheaper and more common 3D printing materials, such as ABS and Alloy 910, showed resistance to a wide range of chemicals.

Despite these encouraging results, there are several factors regarding the influence of solvent on polymer specimens that must be taken into consideration. Firstly, the use of FFF to prepare test specimens results in a structure composed of multiple layers joined together through adhesive forces. This is in contrast to the bulk network structures formed using conventional polymer processing techniques, which generally provide superior mechanical performance and better resistance to solvents. For example, PET has a bulk tensile strength yield of 47 to $90 \mathrm{MPa}$ [50], where as 3-D printed PET ranged from 27 to $45 \mathrm{MPa}$ [51,52]. This is because of the porous/non-solid nature of FFF [53], which provides pores to reduce the material per given volume for strength and provide more surface area for solvents to contact. It is well established that there is a change in strength and rigidity of polymers resulting from the plasticizing action of sorbed chemicals and/or due to the withdrawal of an added plasticizer by a leaching operation [54]. Both mechanisms reduce the operational life of the polymer component. Secondly, the type and degree of interaction between the solvent and polymer can be varied. The polymer may leach into the surrounding solvent, or alternatively the solvent can swell and subsequently bind to the polymer network. This can lead to potentially detrimental effects on any 3D-printed polymer specimens exposed/immersed within solvent. Namely, these solvents can lead to loss of dimensional stability, material softening, loss of mechanical properties and/or retention of (potential harmful/volatile) solvents. This not only leads to inferior material performance, but may pose a health and safety risk. Thirdly, exposure to solvent may encourage oxidation of the polymer, which in turn can result in discoloration, material degradation and loss of properties. This was evident for some specimens immersed within acids. Finally, environmental stress cracking is a major consideration, given the ability of solvents (in particular, corrosive solvents) to encourage crazing. In certain cases, additives present within the polymer may leach out during solvent immersion, further encouraging crazing. Similarly, exposure to UV and/or ozone may further promote oxidation and subsequent stress cracking. This results in brittleness and loss of mechanical integrity of the specimen. It should be noted that the immersion times of 1 week used in this study are substantially longer than the anticipated application/practical times (generally in the range of 2-20 min ), and that the results presented here present an extreme case of solvent effects. However, if 3D-printed parts are to be used repeatedly across a range of different solvents, these effects must be considered.

Future work is needed to study the level and quality of contaminants that possibly eject from 3-D printed labware used in the wet processing of silicon wafers, and explore post-processing methods to prevent their spread if contaminant levels are significant. Further studies are also required to determine how well 3-D printed objects, tools and containers can be cleaned from particles before they are taken inside a clean room. The results reported here provide a foundation for using appropriate cleaning solutions for specific polymers and applications. Quantifying the contaminant levels is especially important in considering the clean room compatibility of 3-D printed equipment. The fabrication of semiconductor devices is sensitive 
to organic and metal contaminations, ranging from particulates to atomic-level impurities. For example, ppb levels of iron in silicon can be detrimental for solar cell functionality, and submicron-sized particle contaminations resulting from conventional, as well as potentially 3D printed labware [55] can e.g. greatly disrupt the fabrication of features in photolithographybased processing. Even small amounts of contaminants on a Si wafer can irreversibly contaminate high-temperature furnaces during annealing steps, so great care is needed applying unconventional labware in clean room processes. Due to the strict cleanliness requirements, non-colored 3-D printing materials are recommended for all lab equipment, as different colorants can contain metal complexes and other nondisclosed compounds. In the future all such materials ingredients may be provided [56], but for now using uncolored filament is the best way to avoid unknown contaminants. The proposed further studies would open clean rooms to the use of 3-D printed tools and provide academics and research personnel a broad toolkit to perform experiments with freely distributed, affordable, and customizable open source equipment. Additionally, in-depth characterization of solvent immersed polymers to determine the effect and prevalence of polymer leaching, swelling, solvent adsorption, oxidation and stress cracking would provide practical information regarding potential solvent/polymer combinations, maximum exposure times and any potential performance or safety risks. The mechanical performance of the 3-D printed specimens before and after solvent immersion would likewise be of great relevance and use. Furthermore, 3-D printed labware shows potential for reducing the costs associated with sample processing. The handle shown in Figure $2 \mathrm{f}$ can be used to pick up a 3 inch wafer jig, and a similar handle can be purchased commercially for \$56 [57]. The 3-D printed handle has a mass of $41 \mathrm{~g}$, which at $\$ 98 / \mathrm{kg}$ costs $\$ 4.02$, which is more than a $92 \%$ savings following a standard economic analysis [58].

\section{Conclusions}

In this study, the possibility of fabricating customized, chemically resistant labware for semiconductor processing steps using affordable FFF-based 3-D printing was investigated. The results show that the chemical resistance of 3-D printing filaments does not greatly differ from tabularized industrial reference values, which shows that the antioxidants and plasticizers used in commercial filaments do not significantly affect the chemical resistance of the polymers. We demonstrate that affordable commercial 3-D printing materials (many of which were not previously tested) are viable options for making customized and chemically resistant labware for handling semiconductor samples in which possible surface contamination does not impair device functionality. The labware presented, especially the customized wet-processing dipper, shows that customized tools can reduce the risk of damaging the sample during handling by ensuring that such tools are tailor-made for each specific sample size. This is especially the case in research, where sample size can be significantly smaller than a full wafer. Customized lab tools also reduce the need to use excess amounts of chemicals, as there would be no need to prepare large amounts of solutions into chemical tanks in wet benches. This, in turn, reduces the processing costs and the risk of personal injury in laboratories. However, as even filament manufacturers do not accurately know the constituents of their products, the applicability of 3-D printed labware for semiconductor processing needs to be studied further.

\section{Acknowledgements}

This work was partially supported by Fulbright Finland and Aleph Objects. The authors acknowledge the provision of facilities by Aalto University at OtaNano - Micronova 
Nanofabrication Centre. This research utilized equipment and facilities under the joint Aalto University and VTT (Technical Research Centre of Finland) Bioeconomy Infrastructure.

\section{References}

1. Symes, M. D., Kitson, P. J., Yan, J., Richmond, C. J., Cooper, G. J., Bowman, R. W., ... \& Cronin, L. (2012). Integrated 3D-printed reactionware for chemical synthesis and analysis. Nature Chemistry, 4(5), 349-354.

2. Kitson, P. J., Symes, M. D., Dragone, V., \& Cronin, L. (2013). Combining 3D printing and liquid handling to produce user-friendly reactionware for chemical synthesis and purification. Chemical Science, 4(8), 3099-3103.

3. Kitson, P. J., Marshall, R. J., Long, D., Forgan, R. S., \& Cronin, L. (2014). 3D printed high-throughput hydrothermal reactionware for discovery, optimization, and scale-up. Angewandte Chemie International Edition, 53(47), 12723-12728.

4. Kitson, P. J., Glatzel, S., Chen, W., Lin, C. G., Song, Y. F., \& Cronin, L. (2016). 3D printing of versatile reactionware for chemical synthesis. Nature protocols, 11(5), 920936.

5. Erkal, J. L., Selimovic, A., Gross, B. C., Lockwood, S. Y., Walton, E. L., McNamara, S., Martin, R. S., Spence, D. M. (2014) 3D printed microfluidic devices with integrated versatile and reusable electrodes. Lab on a Chip, 14, 2023-2032.

6. Kitson, P. J., Rosnes, M. H., Sans, V., Dragone, V., \& Cronin, L. (2012). Configurable 3D-Printed millifluidic and microfluidic 'lab on a chip'reactionware devices. Lab on $a$ Chip, 12(18), 3267-3271.

7. Morgan, A. J., San Jose, L. H., Jamieson, W. D., Wymant, J. M., Song, B., Stephens, P., ... \& Castell, O. K. (2016). Simple and versatile 3D printed microfluidics using fused filament fabrication. PloS one, 11(4), e0152023.

8. Pearce, J.M.; Anzalone, N.C.; Heldt, C.L. Open-Source Wax RepRap 3-D Printer for Rapid Prototyping Paper-Based Microfluidics. Journal of Laboratory Automation 2016, 21(4):510-516.

9. Bhattacharjee, N., Urrios, A., Kang, S., \& Folch, A. (2016). The upcoming 3D-printing revolution in microfluidics. Lab on a Chip, 16(10), 1720-1742.

10. Da Costa, E.T., Mora, M.F., Willis, P.A., do Lago, C.L., Jiao, H., Garcia C.D. Getting started with open-hardware: development and control of microfluidic devices Electrophoresis, 35 (16) (2014), pp. 2370-2377

11. Dragone, V., Sans, V., Rosnes, M. H., Kitson, P. J., \& Cronin, L. (2013). 3D-printed devices for continuous-flow organic chemistry. Beilstein journal of organic chemistry, 9, 951.

12. Sells, E.; Bailard, S.; Smith, Z.; Bowyer, A.; Olliver, V. 2010. RepRap: The Replicating Rapid Prototyper-Maximizing Customizability by Breeding the Means of Production. Proceedings in the World Conference on Mass Customization and Personalization, Cambridge, MA, USA, 7-10 October 2007.

13. Jones, R.; Haufe, P.; Sells, E.; Iravani, P.; Olliver, V.; Palmer, C.; Bowyer, A. RepRapthe Replicating Rapid Prototyper. Robotica 2011, 29 (01): 177-91.

14. Bowyer, A. 3D Printing and Humanity's First Imperfect Replicator. 3D Printing and Additive Manufacturing 2014, 1 (1): 4-5.

15. Pearce, J. Building Research Equipment with Free, Open-Source Hardware. Science 2012, 337 (3100): 1303-4.

16. Pearce, J. Open-Source Lab: How to Build Your Own Hardware and Reduce Research Costs, 1st ed. Elsevier: Waltham, MA, USA, 2014.

17. Pearce, J.M. Laboratory equipment: cut costs with open-source hardware Nature, 505 (7485) (2014) 618-618 
18. Dhankani, K.; Pearce, J. M. Open Source Laboratory Sample Rotator Mixer and Shaker. HardwareX 2017, 1, 1-12.

19. Trivedi, D. K., \& Pearce, J. M. (2017). Open Source 3-D Printed Nutating Mixer. Applied Sciences, 7(9), 942.

20. Baden, T.; Chagas, A.; Marzullo, T.; Prieto-Godino, L.; Euler, T. Open Laware: 3-D Printing Your Own Lab Equipment. PLoS Biology 2015, 13 (3).

21. Damase, T. R., Stephens, D., Spencer, A., \& Allen, P. B. (2015). Open source and DIY hardware for DNA nanotechnology labs. Journal of Biological Methods, 2(3), e24.

22. Lücking, T. H., Sambale, F., Schnaars, B., Bulnes-Abundis, D., Beutel, S., \& Scheper, T. (2015). 3D-printed individual labware in biosciences by rapid prototyping: In vitro biocompatibility and applications for eukaryotic cell cultures. Engineering in Life Sciences, 15(1), 57-64.

23. Gross, B. C., Erkal, J. L., Lockwood, S. Y., Chen, C., \& Spence, D. M. (2014). Evaluation of 3D printing and its potential impact on biotechnology and the chemical sciences. Anal. Chem., 86 (7) 3240-3253

24. Su, C. K., Hsia, S. C., \& Sun, Y. C. (2014). Three-dimensional printed sample load/inject valves enabling online monitoring of extracellular calcium and zinc ions in living rat brains. Analytica Chimica Acta, 838, 58-63.

25. Anzalone, G. C., Glover, A. G., \& Pearce, J. M. (2013). Open-source colorimeter. Sensors, 13(4), 5338-5346.

26. Kelley, C. D., Krolick, A., Brunner, L., Burklund, A., Kahn, D., Ball, W. P., \& WeberShirk, M. (2014). An affordable open-source turbidimeter. Sensors, 14(4), 7142-7155.

27. Wijnen, B., Anzalone, G.C. and Pearce, J.M. Open-source mobile water quality testing platform. Journal of Water Sanitation and Hygiene for Development 2014, 4(3):532537.

28. Carvalho, M. C., \& Eyre, B. D. (2013). A low cost, easy to build, portable, and universal autosampler for liquids. Methods in Oceanography, 8, 23-32.

29. Wijnen, B., Hunt, E. J., Anzalone, G. C., \& Pearce, J. M. (2014). Open-source syringe pump library. PloS one, 9(9), e107216.

30. Zhang, C., Wijnen, B., \& Pearce, J. M. (2016). Open-source 3-D platform for low-cost scientific instrument ecosystem. Journal of Laboratory Automation, 21(4), 517-525.

31. M. Malonado-Torres, H.F. Lopez-Hernandez, P. Jimenez-Sandoval, R. Winkler J. Proteomics, 15 (1) (2014), pp. 57-64

32. Pearce, J. M. (2015). Return on investment for open source scientific hardware development. Science and Public Policy, 43(2), 192-195.

33. Gedde U. (1999) Polymer Physics, 1st ed., Chapman and Hall.

34. Fried, JR. (2014) Polymer Science and Technology, 3rd ed., Prentice Hall.

35. Franssila, S. (2004) Introduction to Microfabrication, 1st edition, John Wiley \& Sons Ltd, England.

36. Open Science Framework https://osf.io/2vaps/, accessed: May 21, 2018.

37. Williams, K. R., Gupta, K., \& Wasilik, M. (2003). Etch rates for micromachining processing-Part II. Journal of Microelectromechanical Systems 12(6) 761-778.

38. Kim, E., Kumar, A., \& Whitesides, G. M. (1995) Combining Patterned Self-Assembled Monolayers of Alkanethiolates on Gold with Anisotropic Etching of Silicon to Generate Controlled Surface Morphologies. Journal of the Electrochemical Society 142.2: 628-633. 
39. Köllensperger, P. A., Karl, W. J., Ahmad, M. M., Pike, W. T., \& Green, M. (2012). Patterning of platinum (Pt) thin films by chemical wet etching in Aqua Regia. Journal of Micromechanics and Microengineering, 22(6), 067001.

40. Kim, J. K., Lee, J. L., Lee, J. W., Eoi Shin, H., Jo Park, Y., \& Kim, T. (1998). Low resistance $\mathrm{Pd} / \mathrm{Au}$ ohmic contacts to p-type GaN using surface treatment. Applied Physics Letters, 73(20), 2953-2955.

41. Chen, J., \& Brewer, W. D. (2015). Ohmic Contacts on p-GaN. Advanced Electronic Materials, 1(8).

42. Lo, Y. S., Huefner, N. D., Chan, W. S., Dryden, P., Hagenhoff, B., \& Beebe, T. P. (1999). Organic and inorganic contamination on commercial AFM cantilevers. Langmuir, 15(19), 6522-6526.

43. Totir, G. G., Frank, M., Vos, R., Arnauts, S., Bearda, T., Kenis, K., ... \& Mannaert, G. (2007). Post ion-implant photoresist removal via wet chemical cleans combined with physical force pretreatments. ECS Transactions, 11(2), 219-226.

44. Desktop_Makes: Tweezers. 3-D model, available: https://www.thingiverse.com/thing:1783167. Accessed March 7, 2018.

45. Curbell Plastics Inc. NY, USA: Chemical resistance chart. Available: https://www.curbellplastics.com/Research-Solutions/Technical-Resources/TechnicalResources/Chemical-Resistance-Chart. Accessed March $7^{\text {th }} 2018$.

46. Sirmax S.p.A., Italy: Chemical resistance of polymers. Available: http://www.sirmax.it/sites/default/files/Chemical\%20resistance\%20of\%20polymers.pdf. Accessed March 7, 2018.

47. Thermo Fisher Scientific Inc. MA, USA: Labware Chemical Compatibility Guide. Available: https://tools.thermofisher.com/content/sfs/brochures/D20480.pdf. Accessed March 7, 2018.

48. Thermo Fisher Scientific Inc. MA, USA: Nalgene Plastic Labware Chemical Resistance Reference Guide. Available:

http://tools.thermofisher.com/content/sfs/posters/LCD-Chemical-Resistance-GuideGALSPCHEMRESPOSTER-EN.pdf. Accessed March 7, 2018.

49. Plastics International, MN, USA: Chemical Resistance Chart. Available: http://www.plasticsintl.com/plastics chemical resistence chart.html. Accessed March 7, 2018.

50. MatWeb - Overview of materials for Polyethylene Terephthalate (PET), Unreinforced - Available online: http://www.matweb.com/search/DataSheet.aspx? MatGUID=a696bdcdff6f41dd98f8eec3599eaa20 Accessed July 26, 2018.

51. Tanikella, N.G., Wittbrodt, B. and Pearce, J.M. (2017). Tensile strength of commercial polymer materials for fused filament fabrication 3D printing. Additive Manufacturing, 15, pp.40-47.

52. Zander, N.E., Gillan, M. and Lambeth, R.H. (2018). Recycled polyethylene terephthalate as a new FFF feedstock material. Additive Manufacturing, 21, pp.174182.

53. Wittbrodt, B. and Pearce, J.M. (2015). The effects of PLA color on material properties of 3-D printed components. Additive Manufacturing, 8, pp.110-116.

54. Xie, Q., Yeager, D. and Chu, J. (1995). The Effect of Chemicals and Solvents on Plastics-An Engineering Practice Guide (No. 950634). SAE Technical Paper. 
55. Pasanen, T. P., von Gastrow, G., Heikkinen, I. T. S., Vähänissi, V., Savin, H., Pearce, J.M. Compatibility of 3-D Printed Devices in Cleanroom Environments for Semiconductor Processing, to be published in 2018.

56. Pearce, J. M. (2018). Expanding the Consumer Bill of Rights for material ingredients. Materials Today, 21(3), 197-198.

57. AS ONE Corporation, Japan: Handle H For 3 Inches HS-30 and others. Available: https://axel-search-e.as-1.co.jp/asone/global/g/NCG157104/?cfrom=I0050000. Accessed March 9, 2018.

58. Wittbrodt, B. T., Glover, A. G., Laureto, J., Anzalone, G. C., Oppliger, D., Irwin, J. L. and Pearce, J. M. Life-cycle economic analysis of distributed manufacturing with open-source 3-D printers. Mechatronics, 2013, 23:713-726. 